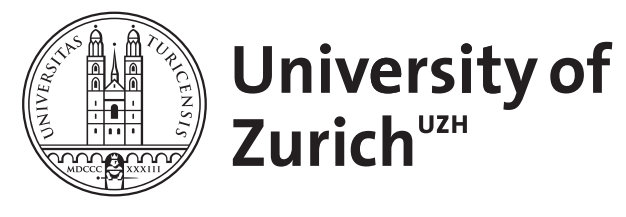

\title{
Clostridium difficile assoziierter Durchfall
}

\author{
Rampini, S K ; Lüthi, B ; Ruef, C ; Speck, R F
}

\begin{abstract}
Zusammenfassung: Die Clostridium difficile assoziierte Diarrhö (CDAD) wird häufig als lästige und relativ banale Nebenwirkung einer Antibiotikatherapie angesehen. Während schon in den 1980er und 90er Jahren Morbidität, Mortalität und entsprechend auch die Kosten von CDAD beträchtlich waren, sind diese aufgrund des Aufkommens eines hochvirulenten Stamms von Clostridium difficile (C.difficile) Anfang2000 deutlich angestiegen. Die pathogenetischen Schlüsselereignisse sind Veränderungen der Darmflora nach Antibiotikagabe, Kolonisation mit einem toxinbildenden C.difficile und dessen intraluminale Vermehrung. Die Therapie besteht bei milden Formen im Absetzen der angeschuldigten Medikamente, bei mäßig bis schwer ausgeprägten Erkrankungen erfolgt die Gabe von Metronidazol oder Vancomycin per os. Als ultima ratio bei toxischem Megakolon muss die subtotale Hemikolektomie erwogen werden. Der verantwortungsvolle Einsatz von Antibiotika ("antibiotic stewardship") in Kombination mit spitalhygienischen Maßnahmen sind essenziell, um Ausbrüchen vorzubeugen und sie einzudämmen
\end{abstract}

DOI: https://doi.org/10.1007/s11377-007-0077-6

Posted at the Zurich Open Repository and Archive, University of Zurich

ZORA URL: https://doi.org/10.5167/uzh-156706

Journal Article

Published Version

Originally published at:

Rampini, S K; Lüthi, B; Ruef, C; Speck, R F (2007). Clostridium difficile assoziierter Durchfall. Der Gastroenterologe, 2(3):170-178.

DOI: https://doi.org/10.1007/s11377-007-0077-6 
Gastroenterologe 2007 $\cdot 2: 170-178$

DOI 10.1007/s11377-007-0077-6

Online publiziert: 8. März 2007

(c) Springer Medizin Verlag 2007

Schwerpunktherausgeber

M. Fried, Zürich
S.K. Rampini • B. Lüthi · C. Ruef · R.F. Speck

Klinik für Infektionskrankheiten und Spitalhygiene,

Departement Innere Medizin, Universitätsspital Zürich

\section{Clostridium difficile assoziierter Durchfall}

\section{„An emerging infection"}

Clostridium difficile ist die häufigste Ursache von nosokomialen Durchfällen. Der C. difficile assoziierten Diarrhö (CDAD) liegt eine pathologisch veränderte Darmflora zu Grunde, gefolgt von einer Exposition oder einem Überwachsen der vorhandenen $C$. difficile mit einem toxinbildenden C. difficile. Ursächlich für das klinische Syndrom der CDAD sind die Toxine $A$ und $B$, etablierte Risikofaktoren sind ein Alter >65 Jahre, Krankenhausaufenthalt und Antibiotikagabe. Die häufigste Manifestation ist eine wässrige Diarrhö, subfebrile Temperaturen und Abdominalkrämpfe, die nach Absetzen der entsprechenden Antibiotika oder nach Gabe von Metronidazol oder Vancomycin meist rasch abheilen. Die Mortalität wurde vor 2003 noch auf ca. 5\% geschätzt. In den letzten 4 Jahren grassiert ein neuer Stamm von C. difficile, welcher eine erhöhte Virulenz im Vergleich zu den „,herkömmlichen“ Stämmen aufweist und mit deutlich erhöhter Morbidität und Mortalität einhergeht.

J.G. Bartlett identifizierte C. difficile als verantwortlichen Erreger der antibiotikaassoziierten pseudomembranösen Kolitis [5]. C. difficile ist ein anaerobes, toxinproduzierendes, grampositives Stäbchen mit der Fähigkeit, Sporen zu bilden. Bei 1-3\% der Bevölkerung wird C. difficile im Stuhl gefunden. Diese asymptomatischen Träger wie auch eine mit Sporen kontaminierte Umgebung, speziell im Krankenhaus, stellen das Reservoir für die Weiterverbreitung dieses Keimes dar.

Der C. difficile assoziierte Durchfall (- Tab.1) wird häufig als relativ harmlose Nebenwirkung einer Antibiotikatherapie beurteilt. Die Morbidität, Mortalität wie auch die anfallenden Kosten widersprechen jedoch dieser oberflächlichen Anschauung: tatsächlich führt eine CDAD bei einer beträchtlichen Zahl der Patienten zum Tod [30]. Daten aus England schätzen die Kosten für ein mittelgroßes Distriktkrankenhaus auf $400.000 \mathrm{E}$, was ca. 2100 verlorenen Hospitalisationstagen entspricht [43]. Vermehrte Aufmerksamkeit hat die CDAD durch das Auftreten eines neuen, hochvirulenten Stamms von C. difficile auch in der Laienpresse erfahren. In diesem Aufsatz möchten wir eine prägnante Übersicht über die Pathogenese, Klinik und Therapie der CDAD und speziell einen kurzen Abriss über diesen neuen virulenten Clostridium-difficileStamm geben.

\section{Pathogenese und Klinik}

C. difficile ist ein grampositives Stäbchen, das sich in anaerober Umgebung vermehrt. Das Bakterium hat die Fähigkeit, bei ungünstigen Lebensbedingungen Endosporen zu bilden, insbesondere wenn es einem aeroben Milieu ausgesetzt wird. Sporen überdauern Jahre in der Umgebung. Das Reservoir von C. difficile sind kolonisierte oder infizierte Patienten und kontaminierte Umgebung. Die Ansteckung mit C. difficile geschieht entweder über die orale Aufnahme von Sporen aus der Umgebung oder über kontaminierte Hände des Medizinpersonals. Nach Aufnahme der Sporen kommt es zur Ausbildung von vegetativen Formen.

Die Wahrscheinlichkeit, mit C. difficile kolonisiert zu werden, steigt mit der Dauer des Krankenhausaufenthalts kontinuierlich an und erhöht sich von $1 \%$ Besiedelung bei einer Dauer von weniger als einer Woche bis auf $50 \%$ bei einer Dauer von mehr als 4 Wochen [16]. Nur in einem Drittel aller kolonisierten Patienten produziert C. difficile Toxine, die Durchfall verursachen [24]. Bei Tieren ist C. difficile auch ein relevantes Pathogen $[6,32]$. Ob Tiere allerdings ein mögliches Erregerreservoir für die fäkal-orale Transmission zum Menschen darstellen, ist bislang nicht ausreichend geklärt.

Die 3 Schlüsselereignisse für eine CDAD sind:

\section{Tab. 1 Definition der Clostridium difficile assoziierten Diarrhö}

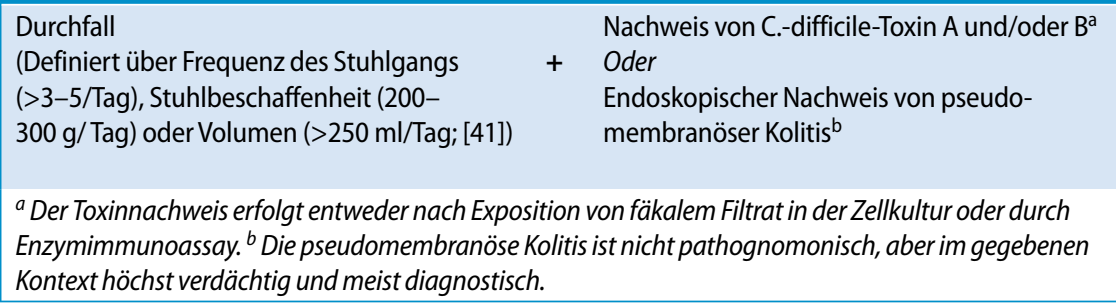


Hier steht eine Anzeige.

算 Springer 


\section{Hier steht eine Anzeige.}

\author{
Springer
}

1. Veränderung der normalen Darmflora

2. Besiedlung des Kolons mit einem toxinbildenden C. difficile

3. Vermehrung mit Toxinbildung

Eine veränderte Darmflora begünstigt das Wachstum von C. difficile [12]. Alle Antibiotika führen zu einer transienten Änderung der physiologischen Darmflora und sind entsprechend der Hauptrisikofaktor für eine CDAD. Dabei ist hervorzuheben, dass auch eine bis 2 Monate zurückliegende Antibiotikagabe zu einer CDAD prädisponiert. Weitere Risikofaktoren sind Krankenhausaufenthalt und fortgeschrittenes Alter [20]. Das Risiko, an einer CDAD zu erkranken, ist im Alter $>65$ Jahren 20-mal höher als im Alter $<20$ Jahren [19]. Immunsuppression, viszeralchirurgische Eingriffe oder andere gastrointestinale Eingriffe scheinen ebenfalls zu einer erhöhten Infektionsrate beizutragen [3]. Die pathogenetische Bedeutung von Protonenpumpenblockern für eine CDAD wird kontrovers diskutiert [10].

Eine physiologische endogene Darmflora ist der beste Schutz gegen eine CDAD.

\section{Auslösende Antibiotika}

Es mag bei erstem Augenschein widersprüchlich erscheinen, dass auch Antibiotika wie z. B. Meropenem oder Piperacillin/Tazobactam [8] mit hervorragender antimikrobieller Aktivität gegenüber C. difficile in vitro zu diesem Krankheitsbild führen können. Die Pathogenese der CDAD beruht auf einer rein intraluminalen Vermehrung von C. difficile, es kommt zu keiner Gewebeinvasion. Die klinischen Manifestationen werden durch die Virulenzfaktoren, insbesondere das Toxin A oder B, hervorgerufen. Antibiotika, welche nicht oder in ungenügender Menge in den Darm ausgeschieden werden, können folglich keine Wirkung gegen C. difficile entfalten - dies trifft auch auf das oben erwähnte Meropenem und Piperacillin/Tazobactam zu. Die verabreichten Antibiotika können jedoch trotzdem zu einer Veränderung der physiologischen Darmflora führen, was das Schlüsselereignis für die Pathogenese der CDAD darstellt.

\section{- Alle Antibiotika können zu einer CDAD führen.}

Ampicillin, Cephalosporine und Clindamycin sind die am häufigsten angeschuldigten Antibiotika, welche zu einer CDAD führen [12]. Antibiotika mit engem Spektrum und Anti-Pseudomonas-Antibiotika scheinen ein geringeres Potenzial zu haben, eine CDAD zu verursachen. In einer Arbeit, welche die Häufigkeit von C. difficile in Stuhlproben in der Hausarztpraxis untersuchte, kamen die Autoren zum Schluss, dass die CDAD eine häufige Ursache der Diarrhö auch beim ambulanten Patienten ist [33]. Amoxicillin, Tetrazykline und Co-trimoxazol waren die angeschuldigten Medikamente. Fluorchinolone schienen trotz ihres weit verbreiteten Gebrauch selten zu einer CDAD zu prädisponieren [12].

Anfang 2000 kam es in Kanada zu einer deutlich erhöhten Inzidenz von CDAD [29]. Epidemiologische Untersuchungen fanden einen Zusammenhang mit dem Auftreten eines neuen C.-difficile-Stamms und dem Verbrauch von Fluorchinolonen ([21, 25, 39]; s. unten).

\section{Toxinproduktion}

Patienten, die über längere Zeit mit C. difficile kolonisiert sind, scheinen ein geringeres Risiko zu haben, eine CDAD zu entwickeln als Kontrollpatienten [31]. Dies mag entweder auf der Besiedlung durch nicht-pathogene Stämme (= Stämme, die keine Toxine bilden) oder auf der erfolgreichen Antikörperbildung gegenüber den Toxinen A und B beruhen [23]. Tatsächlich besteht eine Korrelation zwischen dem Antikörpertiter und der Empfindlichkeit eines Patienten an CDAD zu erkranken. Inwiefern gewisse Antibiotika die Toxinbildung anregen können, bleibt vorderhand unklar.

Die Virulenzfaktoren von C. difficile sind das Toxin A und B. Während sich Toxin A und B in vitro und in Tierexperimenten hinsichtlich ihrer Toxizität deutlich unterscheiden, führen beide Toxine beim Menschen zu intestinalen Schleimhautschädigungen. Es sind auch CDAD beschrieben, die rein durch das Toxin B verursacht sind. 
Klinische Erkrankung und das C.-difficile-Toxin treten fast ausschließlich bei $\mathrm{Pa}$ tienten nach/unter Antibiotikagabe auf.

\section{Klinische Manifestationen}

Der klinische Schweregrad einer CDAD reicht von einer harmlosen Diarrhö, über eine pseudomembranöse Kolitis, Ileus und Perforation bis zum toxischen Megakolon [38]. Die pseudomembranöse Kolitis deutet auf ein fortgeschrittenes Stadium der CDAD hin. Obwohl sie unspezifisch ist, ist der endoskopische Nachweis einer pseudomembranösen Kolitis im Kontext als diagnostisch zu werten. Ileus und toxisches Megakolon sind eine Folge einer ungebremsten Entzündungsreaktion [38]. Die Erkrankung ist fast immer auf das Kolon beschränkt. Das toxische C. difficile assoziierte Megakolon hat eine Mortalität von 24-38\% [11, 31]. Abdominalkrämpfe, Fieber und Leukozytose sind Ausdruck der inflammatorischen Reaktion. Bei schweren und protrahierten Verläufen kann es zu einem intestinalen Proteinverlust („protein-loosing enteropathy“), einer Hypoalbuminämie und Anasarka kommen.

Bei Patienten mit Morbus Crohn oder Colitis ulcerosa deutet die hohe Prävalenz von Darminfektionen (ca. 10\%) und im speziellen von toxinproduzierenden C.-difficile-Infektionen (5,5\%) bei einem Rückfall auf einen pathogenetischen $\mathrm{Zu}$ sammenhang hin [28]. Eine antibiotische Therapie scheint bei dieser Untergruppe den Verlauf günstig zu beeinflussen und sollte bei dieser Untergruppe von Patienten mit Darminfektionen erwogen werden.

Dank der Sensibilisierung der Ärzteschaft gegenüber antibiotikaassoziierten Durchfällen werden CDAD meist schon bei milder Klinik diagnostiziert und schwere Verläufe verhindert. Klinisch kann eine CDAD von Diarrhöen anderer Genese nicht abgegrenzt werden, die Differenzialdiagnose ist je nach Grundkrankheit sehr breit $[14,42]$.

\section{Neue Epidemie}

Anfang der 2oooer Jahre notierte man einen 4-fachen Anstieg von CDAD in Kanada für die Gesamtbevölkerung und insbesondere einen 10-fachen Anstieg für $\mathrm{Pa}$ -

Gastroenterologe 2007 ·2:170-178 DOI 10.1007/s11377-007-0077-6

(c) Springer Medizin Verlag 2007

S.K. Rampini · B. Lüthi · C. Ruef · R.F. Speck Clostridium difficile assoziierter Durchfall. „An emerging infection“

Zusammenfassung

Die Clostridium difficile assoziierte Diarrhö (CDAD) wird häufig als lästige und relativ banale Nebenwirkung einer Antibiotikatherapie angesehen. Während schon in den 1980er und 90er Jahren Morbidität, Mortalität und entsprechend auch die Kosten von CDAD beträchtlich waren, sind diese aufgrund des Aufkommens eines hochvirulenten Stamms von Clostridium difficile Anfang 2000 deutlich angestiegen. Die pathogenetischen Schlüsselereignisse sind Veränderungen der Darmflora nach Antibiotikagabe, Kolonisation mit einem toxinbildenden C. difficile und dessen intraluminale Vermehrung. Die Therapie besteht bei milden Formen im Absetzen der angeschuldigten Medi- kamente, bei mäßig bis schwer ausgeprägten Erkrankungen erfolgt die Gabe von Metronidazol oder Vancomycin per os. Als ultima ratio bei toxischem Megakolon muss die subtotale Hemikolektomie erwogen werden. Der verantwortungsvolle Einsatz von Antibiotika („, antibiotic stewardship") in Kombination mit spitalhygienischen Maßnahmen sind essenziell, um Ausbrüchen vorzubeugen und sie einzudämmen.

Schlüsselwörter

Diarrhö · Clostridium difficile · Ribotyp 027 .

Toxisches Megakolon · Pseudomembranöse Kolitis

\section{Clostridium difficile associated diarrhea. "An emerging infection"}

\section{Abstract}

Clostridium difficile associated diarrhea (CDAD) is a well known side effect of antibiotic therapy. Morbidity, mortality and consequently costs have been dramatically increasing over the last years due to the emergence of a new strain of $C$. difficile. The key pathogenic events are changes in the intestinal flora subsequent to antibiotics, colonization with a toxicogenic strain and its intestinal growth. Mild manifestations only need interruption of the inciting drugs, more severe manifestations must be treated with metronidazol or vancomycin per os. CDAD refractory to treatment may result in toxic megacolon which requires subtotal colectomy. Antibiotic stewardship in combination with infection control are essential to prevent outbreaks of CDAD and if an outbreak occurs to manage it.

\section{Keywords}

Diarrhea - Clostridium difficile - Ribotype 027 . Toxic megacolon · Pseudomembranous colitis 


\section{Schwerpunkt: Akute gastrointestinale Infektionen}
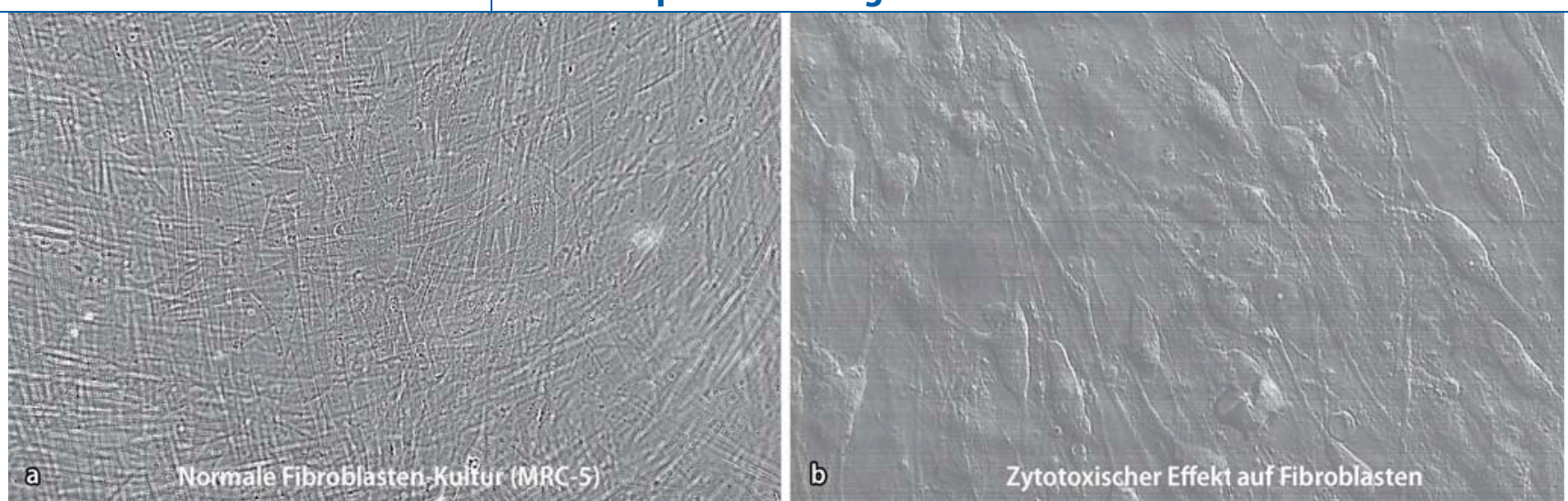

Abb. $1 \Delta$ Goldstandard für die Diagnostik der Clostridium difficile assoziierten Diarrhö ist der Toxinnachweis aus fäkalem Filtrat in der Zellkultur aufgrund des zytotoxischen Effekts. (Die Fotographien wurden freundlicherweise von Herrn PD Dr. R. Zbinden, Institut für Medizinische Mikrobiologie der Universität Zürich, zur Verfügung gestellt)

tienten $>65$ Jahre [19]. Besonders besorgniserregend war, dass die Erkrankung einen schwereren Verlauf zeigte und weniger gut auf eine Therapie ansprach, was sich schließlich auch in einer deutlich erhöhten Zahl von toxischem Megakolon, Notwendigkeit einer Kolektomie, Schock und Tod niederschlug [21, 29]. Im Vergleich zu einer Mortalitätsrate von 4,7\% in der Zeitspanne 1991-92, betrug die Mortalität dieser neuen Epidemie dramatische 13,8\% im Jahr 2003 [30]. Ähnliche Beobachtungen wurden auch an mehreren Institutionen in den USA und Europa gemacht ([3]; www.eurosurveillance.org).

\section{Neuer Clostridiumstamm}

Die Aufarbeitung dieser Ausbrüche ergab, dass ein bis dahin seltener C.-difficileStamm ursächlich ist. Dieser Stamm wird gemäß Methodologie, die zum Nachweis angewendet wird, entweder als NAP1, BI, Toxinotyp III oder Ribotyp o27 bezeichnet [3]. Die durch diesen Stamm verursachte Klinik unterscheidet sich außer durch ihre deutlich schwerere Ausprägung nicht von den bekannten Symptomen einer CDAD. Eine Frühdiagnose ermöglicht sofortige therapeutische Maßnahmen einzuleiten und allfälligen Komplikationen effizient entgegenzutreten [36].

Die ausgeprägte Diarrhö mag auch ursächlich für die erfolgreiche Weiterverbreitung dieses Keims im Krankenhaus sein [39]. Molekularbiologisch zeichnet sich dieser neue Stamm durch die beinahe uniforme Präsenz von folgenden Virulenzfaktoren aus:
- Toxin A und B

- Toxinotyp III

- binäres Toxin

- Fluorchinolonresistenzfaktor

Darüber hinaus hat dieser Stamm eine Deletion eines Locus tcdC, welche für die Herunterregulation der Toxinproduktion bei anderen C. difficile verantwortlich ist. In der Tat produziert dieser Stamm erhöhte Mengen an Toxin A und B [39]. C.-difficile-Stämme, welche vor 2001 gesammelt wurden, zeigen keinen Fluorchinolonresistenzfaktor. Dieser Faktor mag die Assoziation zwischen Fluorchinolongebrauch und dem Ausbruch dieser neuen Epidemie erklären.

\section{Diagnostik}

Goldstandard für die Diagnostik der CDAD ist der Toxinnachweis aus fäkalem Filtrat in der Zellkultur (• Abb. 1). Die meisten verwendeten Tests beruhen auf einem Enzymimmunoassay (EIA) für den Nachweis von Toxin A oder Toxin A und B. Vorteil dieser EIA ist die einfache Handhabung, Verfügbarkeit und Kosten. Die Sensitivität der EIA wird mit 55-90\% und die Spezifität mit $80-95 \%$ angegeben [22]. EIA, die nur das Toxin A nachweisen, werden eine Subpopulation von C.difficile-Stämmen verpassen, die nur das Toxin B produzieren [17]. Diese Stämme sind ebenso pathogen wie Stämme, die Toxin A und B produzieren.

Das C.-difficile-Toxin ist wenig stabil. Bei Raumtemperatur wird das Toxin innerhalb von $2 \mathrm{~h}$ nach Erhalt der Stuhlpro- be degradiert. Falsch-negative Resultate können von einer verzögerten Verarbeitung herrühren oder durch die fehlende Kühlung des Stuhls bis zur Verarbeitung verursacht sein.

Alternative Nachweismethoden beruhen auf dem kulturellen oder PCR-basierten Nachweis von C. difficile. Die Interpretation eines positiven Tests ist jedoch insofern schwierig, da Patienten mit der Länge des Krankenhausaufenthalts eine deutlich erhöhte Kolonisationsrate aufweisen, ohne notwendigerweise an einer CDAD zu erkranken. Hier sollte nach positivem Testergebnis unbedingt untersucht werden, ob der betreffende Patient auch das Toxin A oder B ausscheidet. Bei fehlender Ausscheidung muss an alternative Differenzialdiagnosen gedacht werden.

\section{- Die Untersuchung mittels Kultur und Toxinnachweis ist optimal für die Diagnostik einer CDAD.}

Man geht häufig davon aus, dass ein bis zu 3-mal wiederholtes Testen des Stuhls die Sensitivität maßgeblich erhöht, weshalb dieses Vorgehen häufig als adäquate Maßnahme postuliert wird. Ob dies seine Berechtigung hat, untersuchte eine Studie, in welcher 474 Stuhlproben von insgesamt 396 Patienten auf C.-difficileToxin mit EIA untersucht wurden [27]. Die Sensitivität des angewendeten Tests war $80-90 \% .78$ Patienten wurden wiederholt getestet, nur ein einziger Patient war bei initial negativem Screening nach Wiederholung des Tests positiv auf das 
Toxin diagnostiziert worden. Die Autoren folgerten daraus, dass ein grundsätzliches Wiederholen bei negativem C.difficile-Toxin aufgrund Kostenüberlegungen nicht angebracht ist. Eine im 2006 publizierte Studie kam jedoch zum Schluss, dass wiederholte Stuhluntersuchungen die Sensitivität von 81 auf $95 \%$ steigern [1]. Ein Vergleich verschiedener Studien wird wegen unterschiedlicher Methodik, Patientengut und der Definition einer Diarrhö keine definitive Antwort geben, inwieweit wiederholte Testungen sinnvoll sind. Wir sind der Ansicht, dass die Komplexität der aktuellen Erkrankung wie auch die lokalen Erfahrungen für die Notwendigkeit wiederholter Testungen richtungweisend sein sollten.

\section{Endoskopische Diagnostik}

Die pseudomembranöse Kolitis zeichnet sich makroskopisch durch gelbe, $2-8 \mathrm{~mm}$ große, fibrinöse Auflagerungen auf der Dickdarmschleimhaut aus. In einer Studie zeigte sich überraschenderweise die flexible Sigmoidoskopie gegenüber dem Nachweis von Toxin A in einer Untergruppe von Patienten überlegen [15]. Unterschiedliche Gründe wurden für dieses frappante Resultat diskutiert:

- Läsionen durch andere Erreger verursacht (pseudomembranöse Kolitis typisch, aber nicht pathognomonisch für CDAD)

- Degradation des Toxins beim Transport in die Mikrobiologie

- Interferenz des Toxinnachweises mit anderen im Stuhl vorhandenen Substanzen

Auch hier findet sich keine definitive Antwort über den Stellenwert der Endoskopie für die CDAD-Diagnostik in der Literatur. Eine Endoskopie ist auf jeden Fall bei unklarem persistierendem und wiederholt C.-difficile-Toxin negativem Durchfall für die Weiterabklärung angezeigt.

\section{Behandlung}

Die Behandlung einer CDAD richtet sich nach dem Schweregrad der Erkrankung. Idealerweise sollte das Antibiotikum, welches zu einer CDAD prädisponiert, gestoppt werden. Milde Formen ei-

Tab. 2 Therapeutisches Vorgehen bei Clostridium difficile assoziierter Diarrhö (CDAD)

\begin{tabular}{|c|c|}
\hline Schweregrad der CDAD & Therapie \\
\hline Mild & $\begin{array}{l}\text { Sofern möglich, Absetzen der beschuldigten CDAD-induzierenden } \\
\text { Medikamente }^{\mathrm{a}}\end{array}$ \\
\hline \multirow[t]{3}{*}{ Mäßig bis schwer } & Metronidazol: 3-mal 500 mg/Tag p.o. für 10-14 Tage \\
\hline & Vancomycin: 4-mal 125 mg/Tag p.o. für 10-14 Tage \\
\hline & $\begin{array}{l}\text { Alternativen: } \\
\text { - Teicoplanin 2-mal } 100 \mathrm{mg} / \text { Tag p.o. } \\
\text { - (Vancomycin + Rifampicin) } \\
\text { - (Fusidinsäure) } \\
\text { - (Bacitracin) }\end{array}$ \\
\hline \multirow[t]{3}{*}{$\begin{array}{l}\text { Schwerste Formen } \\
\text { (toxisches Megakolon, Ileus) }\end{array}$} & $\begin{array}{l}\text { Vancomycin durch nasogastrische Sonde oder als Einläufe: 4-mal } \\
125 \mathrm{mg} / \mathrm{Tag} \text { mit möglicher Steigerung auf 4-mal } 500 \mathrm{mg} / \mathrm{Tag}\end{array}$ \\
\hline & Metronidazol 3-mal $500 \mathrm{mg} / \mathrm{Tag}$ i.v. \\
\hline & $\begin{array}{l}\text { Sofern keine Besserung innerhalb von } 48-72 \mathrm{~h} \text { subtotale } \\
\text { Kolektomie erwägen }\end{array}$ \\
\hline
\end{tabular}

ner CDAD bedürfen keiner spezifischen Therapie gegen C. difficile. Die CDAD sollte in den meisten Patienten innerhalb weniger Tage auf eine medikamentöse Therapie mit Sistieren des Durchfalls und Entfieberung ansprechen (• Tab. 2). Bei Patienten mit Ileus oder Megakolon, die nicht auf eine konservative Therapie ansprechen, muss eine Kolektomie in Erwägung gezogen werden [36].

Metronidazol und Vancomycin sind beide gleich effektiv in der Therapie einer CDAD. Metronidazol wird als Ersttherapie gehandelt. Die FDA hat einzig Vancomycin per os als Therapie bei einer CDAD zugelassen. Die Richtlinien der „Society for Healthcare Epidemiology“, der „Infectious Diseases Society of America " und des „Center for Disease Control“ jedoch befürworten als Ersttherapie Metronidazol per os. Vancomycin per os wird vom Darm nicht resorbiert und erreicht intraluminale Konzentrationen im Kolon, welche mehr als die 1oofache minimale Hemmkonzentration von C. difficile übersteigt [2]. Im Gegensatz dazu wird Metronidazol beinahe komplett resorbiert und ist im Kolon nur bei Diarrhö detektierbar.

Gewichtige Argumente gegen eine Ersttherapie mit Vancomycin p.o. sind das mögliche Selektionieren von vancomycinresistenten Enterokokken und die anfallenden Medikamentenkosten. Multiple Faktoren tragen jedoch zum Risiko einer Vancomycinresistenz bei, inklusive Anzahl verabreichter Antibiotika, Dauer einer Antibiotikatherapie, Verschreibung von 3.-Generations-Cephalosporinen,
Clindamycin und Imipenem/Cilastatin, Alter des Patienten etc. [13, 34]. Die perorale Einnahme von Vancomycin führt nicht unweigerlich zu einer Bildung vancomycinresistenter Enterokokken.

Patienten, die keine Medikamente per os einnehmen können, sollten idealerweise Vancomycin durch eine nasogastrische Sonde oder als Einlauf verabreicht bekommen oder alternativ mit Metronidazol intravenös behandelt werden. Vanocmycin i.v. hat keine Wirkung, da es nicht in den Darm ausgeschieden wird und somit intraluminal liegende C. difficile nicht erreicht.

\section{Therapie bei Rezidiven}

Rezidive machen eine wiederholte Therapie mit Metronidazol oder Vancomycin notwendig.

Die Rezidivrate von CDAD ist mit ca. $20 \%$ sehr hoch [23]. Rezidive werden auf eine Reinfektion oder auf einen Rückfall zurückgeführt. Die Beschwerden sind identisch wie bei der Erstmanifestation einer CDAD. Rückfälle treten üblicherweise innerhalb der ersten 10 Tage, in einzelnen Fällen bis zu 2 Monate nach Absetzen einer spezifischen Anti-CDAD-Therapie auf [23]. Resistenzbildung auf Metronidazol oder auf Vancomycin wurde bisher als Ursache eines Rezidivs nicht beschrieben [23]. Hingegen wird spekuliert, ob gastrointestinal verbleibende Sporen zu einem Rezidiv führen können. Metronidazol und Vancomycin haben keine Wirksamkeit gegen Sporen. Insbesonde- 


\section{Schwerpunkt: Akute gastrointestinale Infektionen}

Tab. 3 Rezidivtherapie bei Clostridium difficile assoziierter Diarrhö (CDAD)

\begin{tabular}{|c|c|c|}
\hline $\begin{array}{l}\text { Rezidiv } \\
\text { anzahl }\end{array}$ & Therapie & \\
\hline \multirow[t]{2}{*}{ 1. Rezidiv } & $\begin{array}{l}\text { Vermeiden/Absetzen aller Medika- } \\
\text { mente, die eine CDAD auslösen können }\end{array}$ & Siehe $\bullet$ Tab. 2 \\
\hline & $\begin{array}{l}\text { Erneute Behandlung mit Metronidazol } \\
\text { oder Vancomycin }\end{array}$ & Siehe $\bullet$ Tab. 2 \\
\hline 2. Rezidiv ${ }^{\mathrm{a}}$ & $\begin{array}{l}\text { Vancomycin in ausschleichender } \\
\text { Dosierung und anschließend } \\
\text { als Stoßtherapie }\end{array}$ & $\begin{array}{l}\text { - 1. Woche: 4-mal } 125 \text { mg/Tag p.o. } \\
\text { - 2. Woche: 3-mal } 125 \text { mg/Tag p.o. } \\
\text { - 3. Woche: } 125 \text { mg/Tag p.o. } \\
\text { - 4./5. Woche: } 125 \text { mg p.o. jeden 2. Tag } \\
\text { - 6./7. Woche: } 125 \text { mg p.o. jeden 3. Tag }\end{array}$ \\
\hline \multirow[t]{4}{*}{$\begin{array}{l}\text { Optionen } \\
\text { bei weiteren } \\
\text { Rezidiven }^{\mathrm{a}}\end{array}$} & Probiotika & $\begin{array}{l}\text { - Saccharomyces boulardii 2-mal } \\
500 \mathrm{mg} / \text { Tag über } 30 \text { Tage } \\
\text { - Lactobacillus rhamnosus 2-mal } \\
10^{10} \mathrm{CFU} / \mathrm{Tag}\end{array}$ \\
\hline & Immunglobuline & $\begin{array}{l}\text { - } 0,4 \mathrm{~g} / \mathrm{kd} \text { alle } 3 \text { Wochen; Dauer der } \\
\text { Therapie richtet sich nach dem } \\
\text { klinischen Ansprechen }[26,44]\end{array}$ \\
\hline & Fäkale Bakteriotherapie & $\begin{array}{l}\text { - Verabreichung von frischem Stuhl } \\
\text { oder einem Gemisch von lyophilisierten } \\
\text { Kulturen aus } 10 \text { verschiedenen aeroben } \\
\text { und anaeroben Bakterien }\end{array}$ \\
\hline & Cholestyramin & $\begin{array}{l}\text { - 4-mal } 4 \mathrm{~g} / \mathrm{Tag} \text {, insbesondere im An- } \\
\text { schluss an eine antibiotische Therapie } \\
\text { mit Metronidazol oder Vancomycin }\end{array}$ \\
\hline
\end{tabular}

re Patienten, die keine IgG-Antwort gegen Toxin A bilden, sind gefährdet, an Rezidiven zu erkranken [23]. Einer fehlenden Immunantwort liegen wahrscheinlich unspezifische Faktoren, wie fortgeschrittenes Alter, schlechter Ernährungszustand und allgemeine Komorbidität und nicht eine spezifische Immunschwäche zugrunde.

Einzelne Patienten leiden wiederholt unter Rezidiven [23]. Wir würden von weiteren Abklärungen abraten und pragmatisch gemäß Tab. 3 behandeln. Auch bei Rezidiven ist die Hauptstütze der Therapie Metronidazol oder Vancomycin. In einer Studie aus dem Jahr 1985 wurden hartnäckige Rezidive mit einer immer tiefer dosierten Vancomycindosis gefolgt von einer Vancomycinpulstherapie erfolgreich behandelt [37]. Die Idee dahinter beruht darauf, dass sich in den Tagen ohne Vancomycinexposition während der Pulstherapie, die Sporen zu vegetativen Formen ausbilden und dann entsprechend empfindlich auf die nächste Gabe von Vancomycin sind.

Die in - Tab. 3 aufgelisteten Alternativoptionen ergeben entweder kontrover- se Resultate oder sind nicht ausreichend in Studien validiert worden, um ihren Stellenwert für die Therapie der CDAD $\mathrm{zu}$ definieren. Diese Therapieoptionen müssen auf einer individualisierten Basis evaluiert werden. Eine Minorität von Patienten braucht eine länger dauernde Vancomycingabe per os (125 oder 250 mg täglich oder jeden 2. Tag), um die Rezidive der CDAD zu kontrollieren. Metronidazol kann bei längerfristiger Gabe permanente periphere sensorische Neuropathien hervorrufen, weshalb auf diese Option verzichtet werden sollte [23].

Neue Medikamente mögen in nächster Zukunft zur Verfügung stehen, wie z. B. Nitazoxanid oder Tolevamer $[4,23]$.

\section{Krankenhaushygienische Aspekte}

Im Rahmen der krankenhaushygienischen Maßnahmen (s. auch www.cdc. gov/ncidod/dhqp/id_CdiffFAQ_HCP.ht$\mathrm{ml}$ ) wird eine regelmäßige Händedesinfektion mit Alkohol vor und nach jedem Patientenkontakt empfohlen. Dieses Programm hat zu einer deutlichen Reduktion von nosokomialen Infektionen u. a. von Staphylokokken geführt. Da die Sporen von C. difficile resistent gegenüber Alkohol sind, wird empfohlen, die Hände nach Entfernung der Handschuhe mit desinfizierender Seife (z. B. Triclosan o,03\%) und Wasser zu waschen und anschließend zu desinfizieren. Bezüglich der Effektivität des Händewaschens zur Verminderung der Händekontamination mit Sporen liegen keine eindeutigen Daten vor [35].

\section{จ Alkohol hat keine Wirkung gegen Sporen}

Potenziell kontaminierte Räume werden vorzugsweise mit sporozoid wirksamen Desinfektionsmitteln wie z. B. Javel (Hypochlorit) oder aldehydhaltigen Mitteln gereinigt $[18,45]$.

\section{Kontaktisolation}

Pflegebedürftige bzw. stuhlinkontinente Patienten und insbesondere Patienten auf einer Intensivstation, die an einer CDAD erkrankt sind, sollten kontaktisoliert werden. Die Kontaktisolation bei Patienten auf der Intensivstation sollte erst bei negativem Nachweis auf Toxin, welcher 2 Tage nach Ende der adäquaten Antibiotkatherapie durchgeführt wird, aufgehoben werden. Dasselbe trifft auch auf pflegebedürftige Patienten zu, die an einer persistierenden Diarrhö leiden. Bei Sistieren der Diarrhö kann auf den Nachweis verzichtet werden.

Die Kontaktisolation basiert auf der Annahme, dass erkrankte Patienten das Hauptreservoir für den Erreger darstellen und eine Übertragung durch Kontamination der Umgebung bzw. durch die Hände des Medizinpersonals geschieht. Die Effektivität von Kontaktisolationsmaßnahmen konnte mehrfach im Rahmen von Ausbrüchen von CDAD gezeigt werden $[9,45]$. Selbständige Patienten werden auf eine gute Händehygiene aufmerksam gemacht.

Eine Kontaktisolation beinhaltet die Unterbringung in einem Einzelzimmer bis zum Sistieren der Diarrhö, Tragen von Handschuhen und einer Überschürze durch das Medizinalpersonal beim Betreten des Zimmers sowie Händehygiene vor und nach Kontakt mit dem Patienten $[31,35]$. 
Hier steht eine Anzeige.

第 Springer 
Bei fehlender Möglichkeit, erkrankte Personen in einem Einzelzimmer unterzubringen, muss ein Isolationsbereich definiert werden. Untersuchungsgeräte, wie Stethoskop, Thermometer und Blutdruckgerät sollen ausschließlich beim erkrankten Patienten benutzt werden. Der korrekten Aufbereitung von Gegenständen und Instrumenten (z. B. Fieberthermometer), die bei mehreren Patienten zur Anwendung kommen, kommt große Bedeutung zu. Es gibt Untersuchungen, die zeigen, dass die Umgebung (Flächen und Objekte) von Patienten mit CDAD massiv durch C. difficile kontaminiert ist.

\section{„Antibiotic stewardship"}

Verschiedene Studien haben eindeutig gezeigt, dass Ausbrüche von CDAD nur mit einer effizienten Reduktion des Antibiotikagebrauchs in Kombination mit rigorosem Umsetzen der Kontaktisolation kontrolliert werden können. Insbesondere problematisch gestaltet sich ein zu generöser Gebrauch von 3.-GenerationsCephalosporinen und Clindamycin. Hier muss nochmals betont werden, dass Fluorchinolone bis anfangs der 2oooer Jahre als Antibiotika mit nur geringem Risikopotenzial für eine CDAD angesehen wurden. Die besorgniserregenden Ausbrüche mit dem neuen C.-difficileStamm, BI/ NAP1, sind jedoch Beweis, dass Bakterien Virulenzfaktoren erwerben und zu neuen „emerging infections“ mit schweren Krankheitsbildern führen und somit potenziell alle Antibiotika zu dieser Entwicklung beitragen können. Diese Neuentwicklung unterstreicht die Bedeutung eines effizienten „Antibioticstewardship-Programms“ mit Strategien oder Maßnahmen zur Optimierung des Antibiotikaeinsatzes.

\section{Fazit für die Praxis}

\section{Krankenhaushygienische Maßnahmen führen zu einer eindrücklichen Reduktion der Inzidenz von Clostridium difficile as- soziierter Diarrhö [45]. Aufgrund der Bio- logie von C. difficile und aufgrund der oft ungenügenden Disziplin bei diesen hygi- enischen Maßnahmen, insbesondere be- züglich der hygienischen Händedesinfek- tion, werden Ausbrüche mit C. difficile im-}

mer wieder stattfinden. Dies spiegelt sich auch in der kürzlich erschienen Literatur wider, in der der Stellenwert der krankenhaushygienischen Maßnahmen bezüglich des Auftretens des neuen C.-difficileStamms kontrovers diskutiert wird $[7,40]$.

\section{Korrespondierender Autor PD Dr. R.F. Speck}

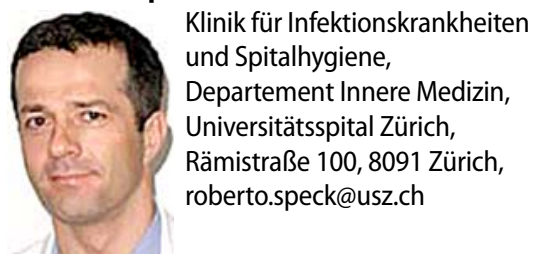

Interessenkonflikt. Es besteht kein Interessenkonflikt. Der korrespondierende Autor versichert, dass keine Verbindungen mit einer Firma, deren Produkt in dem Artikel genannt ist, oder einer Firma, die ein Konkurrenzprodukt vertreibt, bestehen. Die Präsentation des Themas ist unabhängig und die Darstellung der Inhalte produktneutral.

\section{Literatur (Auswahl)}

1. Arango Jl, Restrepo A, Schneider DL et al. (2006) Incidence of Clostridium difficile-associated diarrhea before and after autologous peripheral blood stem cell transplantation for lymphoma and multiple myeloma. Bone Marrow Transplant 37: 517-521

2. Aslam S, Hamill RJ, Musher DM (2005) Treatment of Clostridium difficile-associated disease: old therapies and new strategies. Lancet Infect Dis 5: 549-557

3. Bartlett JG (2006) Narrative review: the new epidemic of Clostridium difficile-associated enteric disease. Ann Intern Med 145: 758-764

4. Bartlett JG (2006) New drugs for Clostridium difficile infection. Clin Infect Dis 43: 428-431

5. Bartlett JG, Chang TW, Gurwith M et al. (1978) Antibiotic-associated pseudomembranous colitis due to toxin-producing clostridia. N Engl J Med 298: 531-534

8. Bourgault AM, Lamothe F, Loo VG, Poirier L (2006) In vitro susceptibility of Clostridium difficile clinical isolates from a multi-institutional outbreak in Southern Quebec, Canada. Antimicrob Agents Chemother 50: 3473-3475

10. Cunningham R (2006) Proton pump inhibitors and the risk of Clostridium difficile-associated disease: further evidence from the community. CMAJ 175: 757

12. Freeman J, Wilcox MH (1999) Antibiotics and Clostridium difficile. Microbes Infect 1:377-384

13. Gerding DN (1997) Is there a relationship between vancomycin-resistant enterococcal infection and Clostridium difficile infection? Clin Infect Dis 25 (Suppl 2): S206-S210

14. Ginsburg PM, Thuluvath PJ (2005) Diarrhea in liver transplant recipients: etiology and management. Liver Transpl 11: 881-890

15. Johal SS, Hammond J, Solomon K et al. (2004) Clostridium difficile associated diarrhoea in hospitalised patients: onset in the community and hospital and role of flexible sigmoidoscopy. Gut 53: 673-677
16. Johnson S, Clabots CR, Linn FV et al. (1990) Nosocomial Clostridium difficile colonisation and disease. Lancet 336: 97-100

17. Johnson S, Sambol SP, Brazier JS et al. (2003) International typing study of toxin A-negative, toxin Bpositive Clostridium difficile variants. J Clin Microbiol 41: 1543-1547

19. Karlstrom O, Fryklund B, Tullus K, Burman LG (1998) A prospective nationwide study of Clostridium difficile-associated diarrhea in Sweden. The Swedish C. difficile Study Group. Clin Infect Dis 26 141-145

20. Lee KS, Shin WG, Jang MK et al. (2006) Who are susceptible to pseudomembranous colitis among patients with presumed antibiotic-associated diarrhea? Dis Colon Rectum 49: 1552-1558

22. Louie TJ (2006) Treatment of first recurrences of Clostridium difficile-associated disease: waiting for new treatment options. Clin Infect Dis 42: 765-767

23. Maroo S, Lamont JT (2006) Recurrent clostridium difficile. Gastroenterology 130: 1311-1316

24. McCoubrey J, Starr J, Martin H, Poxton IR (2003) Clostridium difficile in a geriatric unit: a prospective epidemiological study employing a novel Slayer typing method. J Med Microbiol 52: 573-578

26. McPherson S, Rees CJ, Ellis R et al. (2006) Intravenous immunoglobulin for the treatment of severe, refractory, and recurrent Clostridium difficile diarrhea. Dis Colon Rectum 49: 640-645

27. Mohan SS, McDermott BP, Parchuri S, Cunha BA (2006) Lack of value of repeat stool testing for Clostridium difficile toxin. Am J Med 119: 356 e7-e8

28. Pepin J, Saheb N, Coulombe MA et al. (2005) Emergence of fluoroquinolones as the predominant risk factor for Clostridium difficile-associated diarrhea: a cohort study during an epidemic in Quebec. Clin Infect Dis 41: 1254-1260

29. Pepin J, Valiquette L, Alary ME et al. (2004) Clostridium difficile-associated diarrhea in a region of Quebec from 1991 to 2003: a changing pattern of disease severity. CMAJ 171: 466-472

30. Poutanen SM, Simor AE (2004) Clostridium difficileassociated diarrhea in adults. CMAJ 171:51-58

32. Riley TV, Wetherall F, Bowman J et al. (1991) Diarrheal disease due to Clostridium difficile in general practice. Pathology 23: 346-349

36. Tedesco FJ, Gordon D, Fortson WC (1985) Approach to patients with multiple relapses of antibiotic-associated pseudomembranous colitis. Am J Gastroenterol 80: 867-868

40. Whelan K, Judd PA, Preedy VR, Taylor MA (2004) Enteral feeding: the effect on faecal output, the faecal microflora and SCFA concentrations. Proc Nutr Soc 63: 105-113

41. Wiesen P, Van Gossum A, Preiser JC (2006) Diarrhoea in the critically ill. Curr Opin Crit Care 12: 149154

42. Wilcox MH (1998) Clostridium difficile infection: appendix. J Antimicrob Chemother 41 (Suppl C): 71-72

44. Wilcox MH, Fawley WN (20009 Hospital disinfectants and spore formation by Clostridium difficile. Lancet 356: 1324

45. Zafar AB, Gaydos LA, Furlong WB et al. (1998) Effectiveness of infection control program in controlling nosocomial Clostridium difficile. Am J Infect Control 26: 588-593

\section{Das komplette Literaturverzeichnis ..}

... finden Sie in der elektronischen Version dieses Beitrags unter www.DerGastroenterologe.springer.de 